\title{
The Burden and Trends of Primary Liver Cancer Caused by Specific Etiologies from 1990 to 2017 at the Global, Regional, National, Age, and Sex Level Results from the Global Burden of Disease Study 2017
}

\author{
Longfei Lin ${ }^{a} \quad$ Lei Yan $^{b} \quad$ Yuling Liu ${ }^{a} \quad$ Changhai $\mathrm{Qu}^{\mathrm{c}}$ Jian $\mathrm{Ni}^{\mathrm{c}} \quad \mathrm{Hui} \mathrm{Li}^{\mathrm{a}}$ \\ a Institute of Chinese Materia Medica, China Academy of Chinese Medical Sciences, \\ Beijing, China; ${ }^{b}$ Fengtai District Community Health Center, Beijing, China; ${ }^{c}$ School of \\ Chinese Material Medica, Beijing University of Chinese Medicine, Beijing, China
}

\section{Keywords}

Global Burden of Disease study · Hepatocellular carcinoma - Incidence and death · Hepatitis $B$ virus $\cdot$ Hepatitis $C$ virus

\begin{abstract}
Background: Liver cancer is one of the leading causes of cancer-related deaths worldwide. The primary causes of liver cancer include hepatitis B virus (HBV), hepatitis C virus (HCV), alcohol consumption, nonalcoholic fatty liver disease, and other factors. Aims: The objective of this study was to evaluate the global and sex-, age-, region-, country-, and etiology-related liver cancer burden, as well as the trends in liver cancer caused by different etiologies. Methods: The causes of liver cancer from 1990 to 2017, including global, regional, and national liver cancer incidence, mortality, and etiology, were collected from the Global Burden of Disease study 2017, and the time-dependent change in the trends of liver cancer burden was evaluated by annual percentage change. Results: The global liver cancer incidence and mortality have been increasing. There were 950,000 newly-diagnosed liver cancer cases and over 800,000 deaths in 2017, which is more than twice the numbers recorded in 1990. HBV and HCV are the major causes of liver cancer. HBV is the major risk factor of liver cancer in Asia, while $\mathrm{HCV}$ and alcohol abuse are the major risk factors in the high sociodemographic index and
\end{abstract}

L. Lin and L. Yan contributed equally to this work.

Hui Li

Institute of Chinese Materia Medica, China Academy of Chinese Medical Sciences, Dongcheng District Dongzhimen Nanxiaojie within 16, Beijing 100700 (China) lihuizys@126.com
Jian $\mathrm{N}$

School of Chinese Material Medica, Beijing University of Chinese Medicine, Chaoyang District

Bei San Huan Dong Lu No. 11, Beijing 100029 (China) njtcm@263.net 
high human development index regions. The mean onset age and incidence of liver cancer with different etiologies have gradually increased in the past 30 years. Conclusions: The global incidence is still rising and the causes have national, regional, or population specificities. More targeted prevention strategies must be developed for the different etiologic types in order to reduce liver cancer burden.

(C) 2020 The Author(s)

Published by S. Karger AG, Basel

\section{Introduction}

Primary liver cancer is one of the most common malignancies worldwide. According to the 2018 Global Cancer Statistics, it ranks 7th and 3rd among all cancers in terms of global incidence and mortality, respectively. A total of 840,000 new cases of liver cancer and 780,000 related deaths were recorded in 2018 [1]. In addition, the 5-year relative survival rate of liver cancer patients was 18.4\% (2009-2015) and 14.1\% (2010-2014) in the USA and China, respectively $[2,3]$. The disease burden of liver cancer is one of the key factors responsible for the morbidity among alcoholics.

Primary liver cancer is associated with hepatitis B virus (HBV) infection, hepatitis $\mathrm{C}$ virus (HCV) infection, chronic alcoholism, nonalcoholic fatty liver disease (NAFLD), and other factors (aflatoxin B1, microcystins, and drinking water pollution) [4-6]. Previous studies have shown some geographic heterogeneity in the etiologic factors of liver cancer, which vary across countries and regions. For example, HCV infection and alcoholic liver disease are the main causes of liver cancer in developed countries, whereas HBV infection is the primary risk factor in most developing countries [7, 8].

In this study, we examined the global burden of liver cancer from the Global Burden of Disease (GBD) 2017 study by analyzing the incidence and mortality rates of five major etiologic types of this cancer, as well as the trends in the above across 195 countries or territories from 1990 to 2017 by sex and age [9]. Understanding the geographic, temporal, and demographic distribution of liver cancer incidence and mortality will greatly aid in developing targeted prevention strategies.

\section{Methods}

\section{Data Source}

The annual liver cancer incidence and deaths in groups demarcated by sex, territory, country, age, and etiology as well as the corresponding age-standardized rates were extracted from the GBD study. The data were collected from 195 countries and territories, which were divided into five regions (low, low-medium, medium, high-medium, and high) based on the sociodemographic index (SDI), and also classified as high, upper middle, lower middle, and low according to the World Bank human development index (HDI). In addition, these countries were geographically divided into 21 territories, such as East Asia and Central Asia (Table 1). All cancer-related epidemiological data were obtained from single cancer registries or databases of cancer registries, such as the Cancer Incidence in Five Continents (CI5), Surveillance, Epidemiology, and End Results (SEER), or the Nordic Cancer Registries database (NORDCAN). The disease was classified according to the International Classification of Diseases 9 (ICD-9) and ICD-10, and mapped to the global burden of disease. The ICD-9 disease codes for liver cancer are 155-155.9 and 211.5, and the ICD-10 codes are C22-C22.9 and D13.4. According to the GBD database, the etiology of liver cancer was classified into HBV, HCV, alcoholic liver disease, NAFLD, and other causes [9].

Statistical Analysis

The trends in liver cancer incidence and mortality rates from 1990 to 2017 across the different groups (see above) were determined as age-standardized incidence rate (ASIR) and age-standardized death rate 


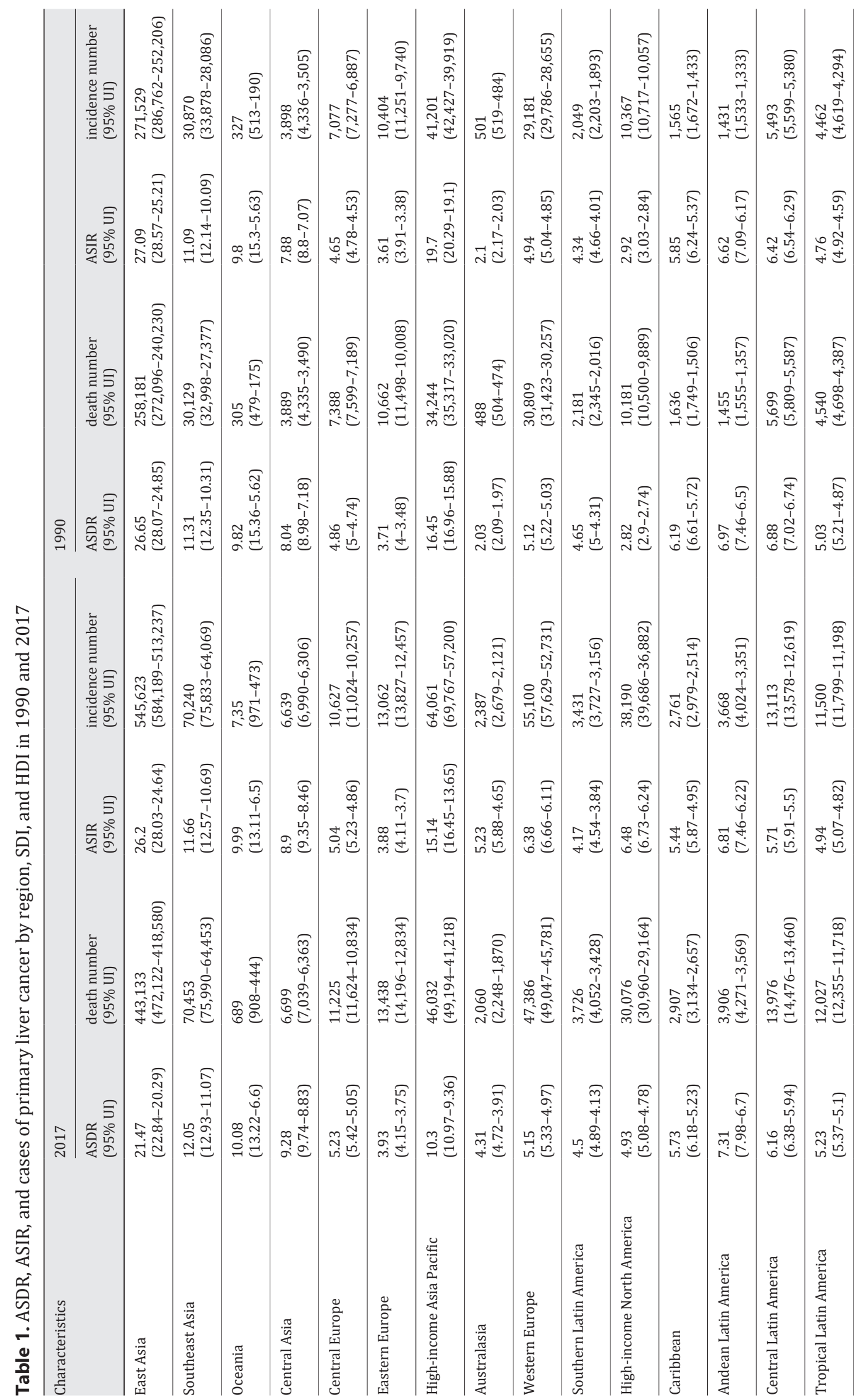




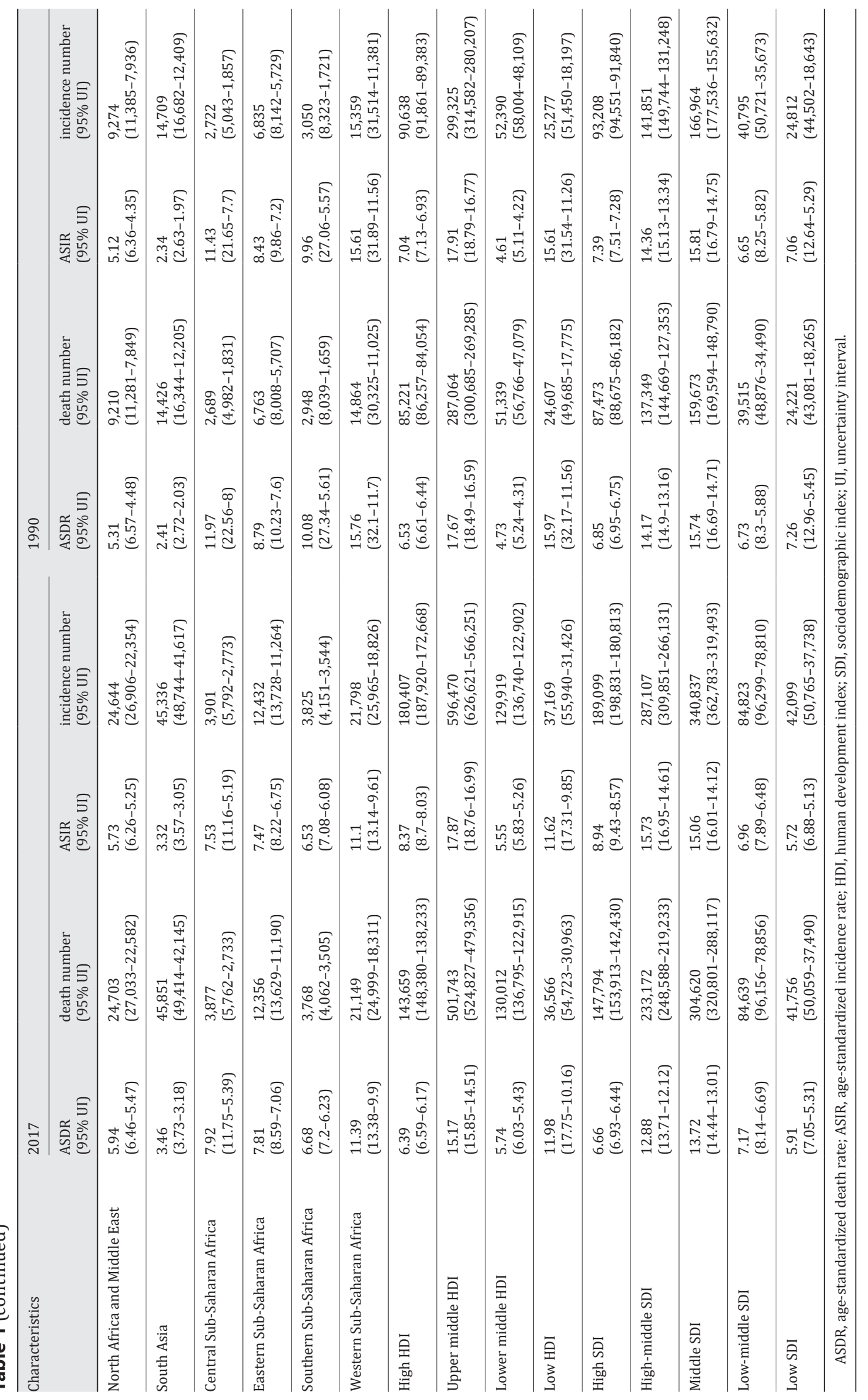


Lin et al.: Global Burden of Primary Liver Cancer in 2017

(ASDR), respectively, in order to eliminate any differences arising from intragroup heterogeneity in sex and age. ASIR was calculated directly (per 100,000 individuals). The numerical of ASDR and ASIR was represented as value (range of $95 \%$ uncertainty interval). The Joinpoint regression model was used, and statistically significant trends were determined using the National Cancer Institute Joinpoint regression program (version 4.1.0). The annual percentage change (APC) in each trend was also calculated [10].

\section{Results}

\section{Global Liver Cancer Burden}

The global liver cancer incidents, deaths, ASIR, and ASDR in various geographic and etiologic groups in 1990 and 2017 are shown in Tables 1 and 2. Over 800,000 (819,435 [789,721-855,505]) liver cancer-related deaths occurred worldwide in 2017, which was nearly twice the number of deaths in 1990 (452,688 [426,298-490,453]). Similarly, $953,076(916,543-997,047)$ new liver cancer cases were diagnosed in 2017, which was again two-fold higher than in 1990. The ASIR also showed an increasing trend over the years. The liver cancer burden also varied according to the income levels of the countries, and was higher in the upper middle (ASDR = 15.17 [14.51-15.85]; ASIR = 17.87 [16.9918.76]) and low (ASDR = 11.98 [10.16-17.75]; ASIR = 11.62 [9.85-17.31]) HDI regions. In contrast, ASDR and ASIR decreased significantly in the low HDI regions and were approximately 25\% lower than in 1990.

Furthermore, the ASIR and ASDR of liver cancer were higher in the high-middle SDI and middle SDI regions compared to the others. The middle SDI regions had the highest liver cancer burden, with an ASDR and ASIR of 13.72 (13.01-14.44) and 15.06 (14.12-16.01), respectively, in 2017. The ASDR in the high SDI regions was lower in 2017 than in 1990, but the ASIR increased by nearly $21 \%$ from 7.39 (7.28-7.51) to 8.94 (8.57-9.43). The ASDR and ASIR in the low SDI regions were 5.91 (5.31-7.05) and 5.72 (5.13-6.88), respectively, in 2017, which was about 19\% lower compared to 1990 and corresponded to the greatest decrease in both factors among all SDI regions. Despite the low ASDR and ASIR in the lowmiddle and low SDI regions in 2017, the ASDR/ASIR ratios were $>1$ in both and similar to 1990. On the other hand, the ASDR/ASIR ratios decreased significantly in the other three regions over the years.

Geographically, East Asia had the highest liver cancer burden, which accounted for $>50 \%$ of the global burden, and its 2017 ASDR and ASIR were 21.47 (20.29-22.84) and 26.2 (24.6428.03), respectively. The 2017 the ASDRs in Southeast Asia, Western Sub-Saharan Africa, high-income Asia Pacific, and Oceania were all >10. Compared to 1990, the ASDR decreased by $>25 \%$ in high-income Asia Pacific, Central Sub-Saharan Africa, Southern Sub-Saharan Africa, and Western Sub-Saharan Africa, but increased by $>40 \%$ in Australasia, high-income North America, and South Asia in 2017.

Etiologically, nearly $39.1 \%$ of liver cancer-related deaths in 2017 were caused by HBV infection. The ASDR of liver cancer due to hepatitis B (LHB) was 3.99 (3.74-5.27) in 2017, which was $14 \%$ lower than in 1990 . Newly-diagnosed LHB cases accounted for $45.6 \%$ of the total LHB cases in 2017, and the ASIR was 4.93 (4.62-5.29). HCV was the second most common cause, accounting for $29.1 \%(234,336$ [219,373-250,559]) of liver cancer-related deaths in 2017. The ASDR and ASIR of liver cancer due to hepatitis C (LHC) were 2.97 (2.793.17 ) and 3.25 (3.04-3.46), respectively, and the latter increased by 30\% compared to 1990 . Alcohol consumption, nonalcoholic steatohepatitis (NASH), and other causes accounted for $16.6,8.4$, and $8.6 \%$, respectively, of the newly-diagnosed liver cancer cases in 2017 . The ASDRs of that year for liver cancer due to alcohol use (LAU), liver cancer due to NASH (LNA), and liver cancer due to other causes (LOC) were 1.61 (1.43-1.84), $0.85(0.75-0.94)$, and 0.79 


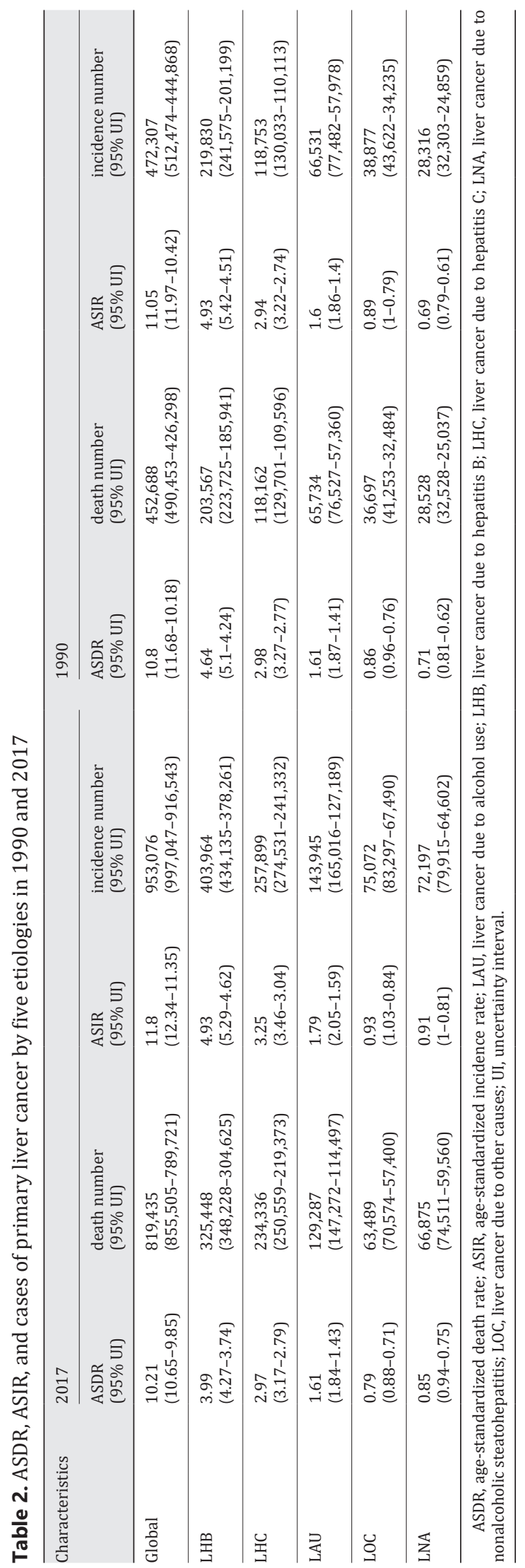




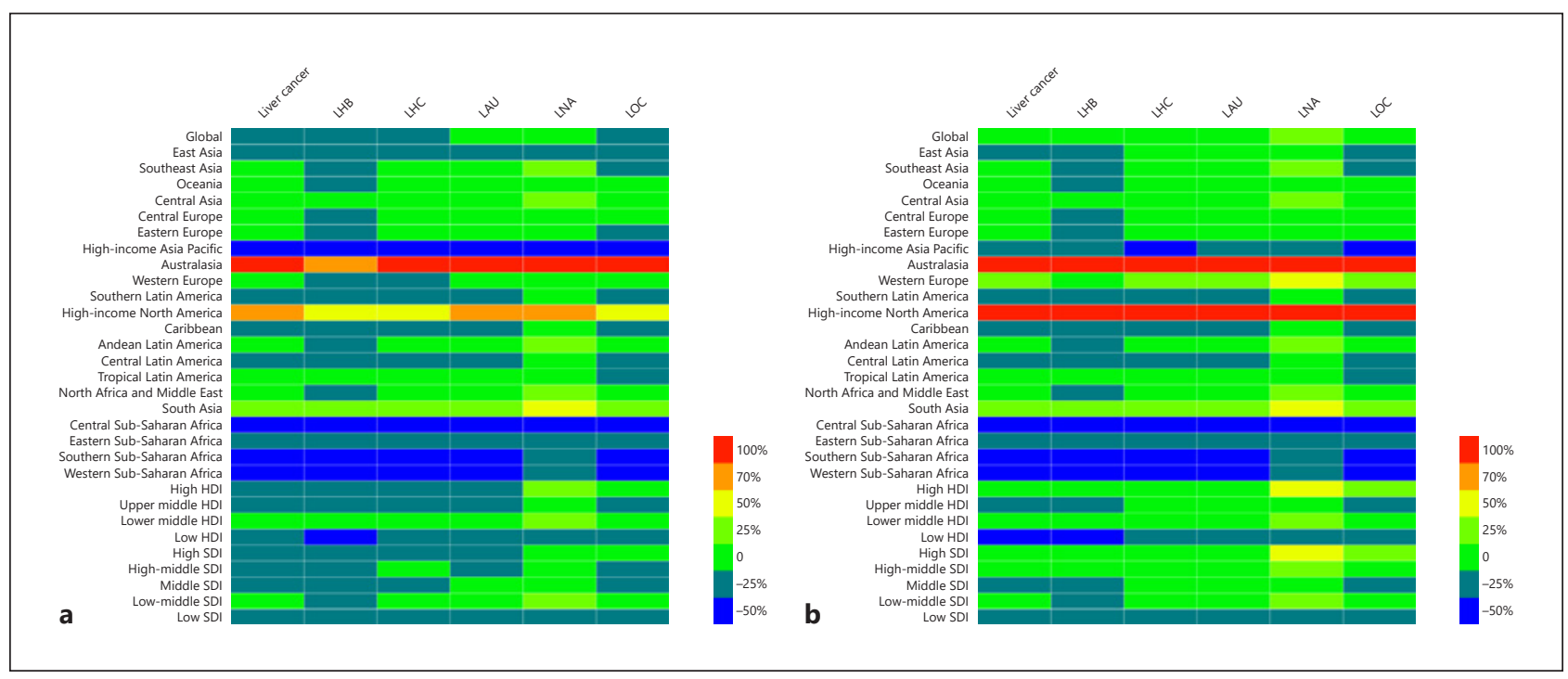

Fig. 1. Relative changes in ASDR (a) and ASIR (b) of liver cancer and five etiologies between 1990 and 2017 in different territories. ASDR, age-standardized death rate; ASIR, age-standardized incidence rate; HDI, human development index; LAU, liver cancer due to alcohol use; LHB, liver cancer due to hepatitis B; LHC, liver cancer due to hepatitis C; LNA, liver cancer due to nonalcoholic steatohepatitis; LOC, liver cancer due to other causes; SDI, sociodemographic index.

(0.71-0.88), respectively. Compared to 1990, the ASDR and ASIR of LNA increased significantly in 2017 by 19.7 and 31.9\%, respectively. The relative changes in ASDR and ASIR of liver cancer and five etiologies between 1990 and 2017 in different territories are shown in Figure 1.

\section{Regional Differences in Liver Burden}

The causes of liver cancer were unique to different regions (as shown in Table 3 and online suppl. Table S1; see www.karger.com/doi/10.1159/000508568 for all online suppl. material). The 2017 ASDR and ASIR of LHB in East Asia were 11.31 (10.55-12.19) and 14.47 (13.47-15.60), respectively, which were the highest among all 21 geographic regions. There were 238,103 (221,878-256,971) LHB-related deaths and 305,347 (283,907-329,708) LHB incidents in this region in 2017, which accounted for 73.2 and $75.6 \%$ of the global LHB burden, respectively. In addition, HBV was the primary cause of liver cancer in East Asia, accounting for 53.7 and $56 \%$ of all liver cancer-related deaths and incidents, respectively, in that region. In contrast, Southern Latin America had the lowest LHB burden with an ASDR and ASIR of $0.23(0.21-0.27)$ and $0.24(0.21-0.27)$, respectively, which accounted for 5 and $5.4 \%$ of all liver cancer-related deaths and incidents, respectively.

The LHC burden was relatively high in East Asia, Southeast Asia, Central Asia, highincome Asia Pacific, and Central Sub-Saharan Africa, with respective ASDRs of 4.90 (4.545.30), 3.38 (2.88-3.86), 3.78 (3.37-4.21), 4.97 (4.29-5.42), and 3.30 (2.14-4.96) in 2017. High-income Asia Pacific had the highest ASIR (6.95 [6.00-7.67]), followed by East Asia (5.49 [5.08-5.94]). LHC-related deaths accounted for $>40 \%$ of all liver cancer-related deaths in high-income Asia Pacific, Western Europe, high-income North America, Central Latin America, Tropical Latin America, North Africa, and the Middle East. Andean Latin America had the lowest ASDR (0.83 [0.71-0.96]) and ASIR (0.73 [0.63-0.84]) of LHC.

Approximately $15 \%$ of the total liver cancer-related deaths and incidents were caused by alcoholism. Southeast Asia had the highest ASDR (3.35 [2.66-4.19]) and ASIR (3.23 [2.57-

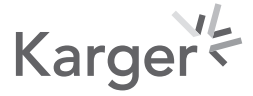




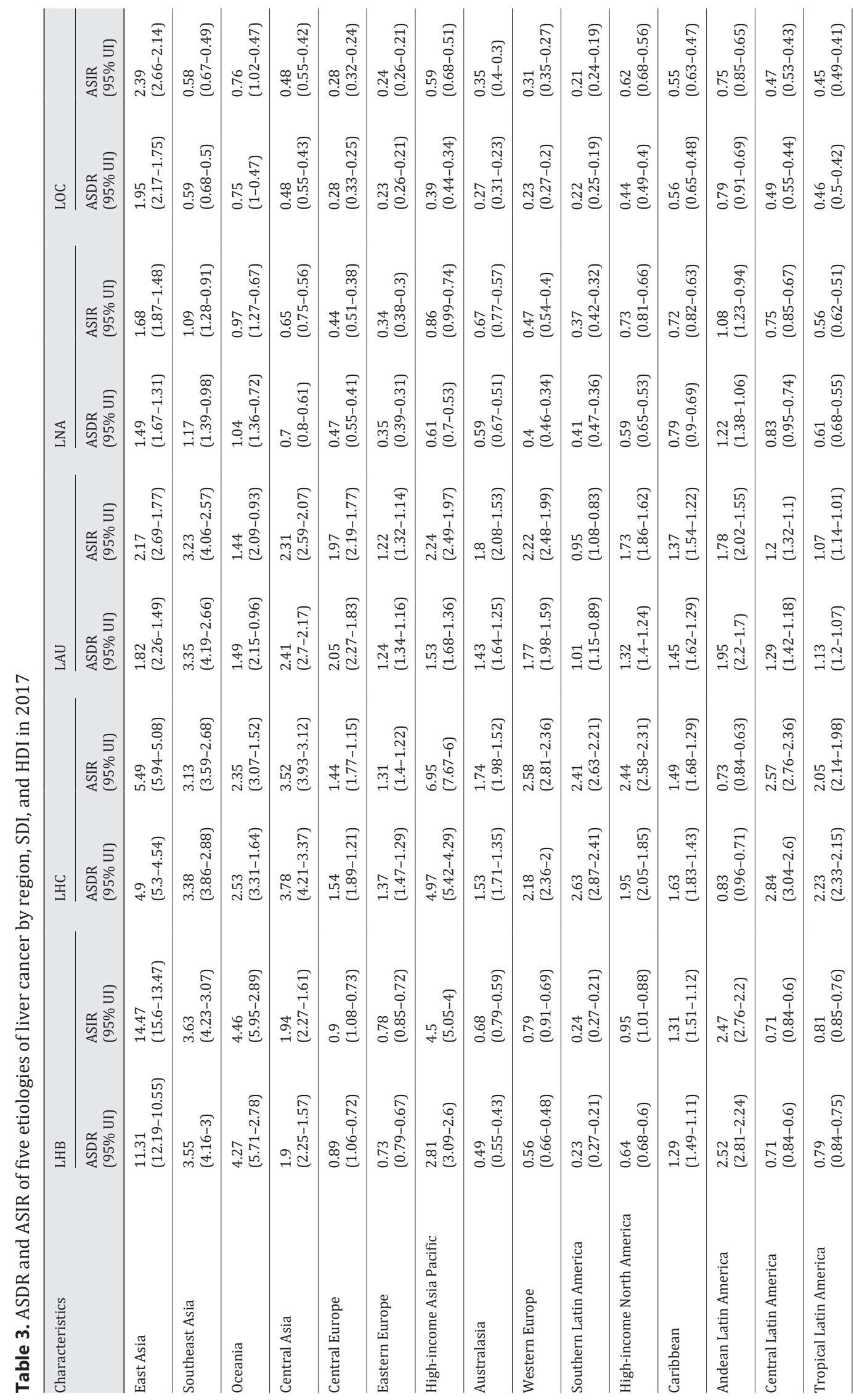




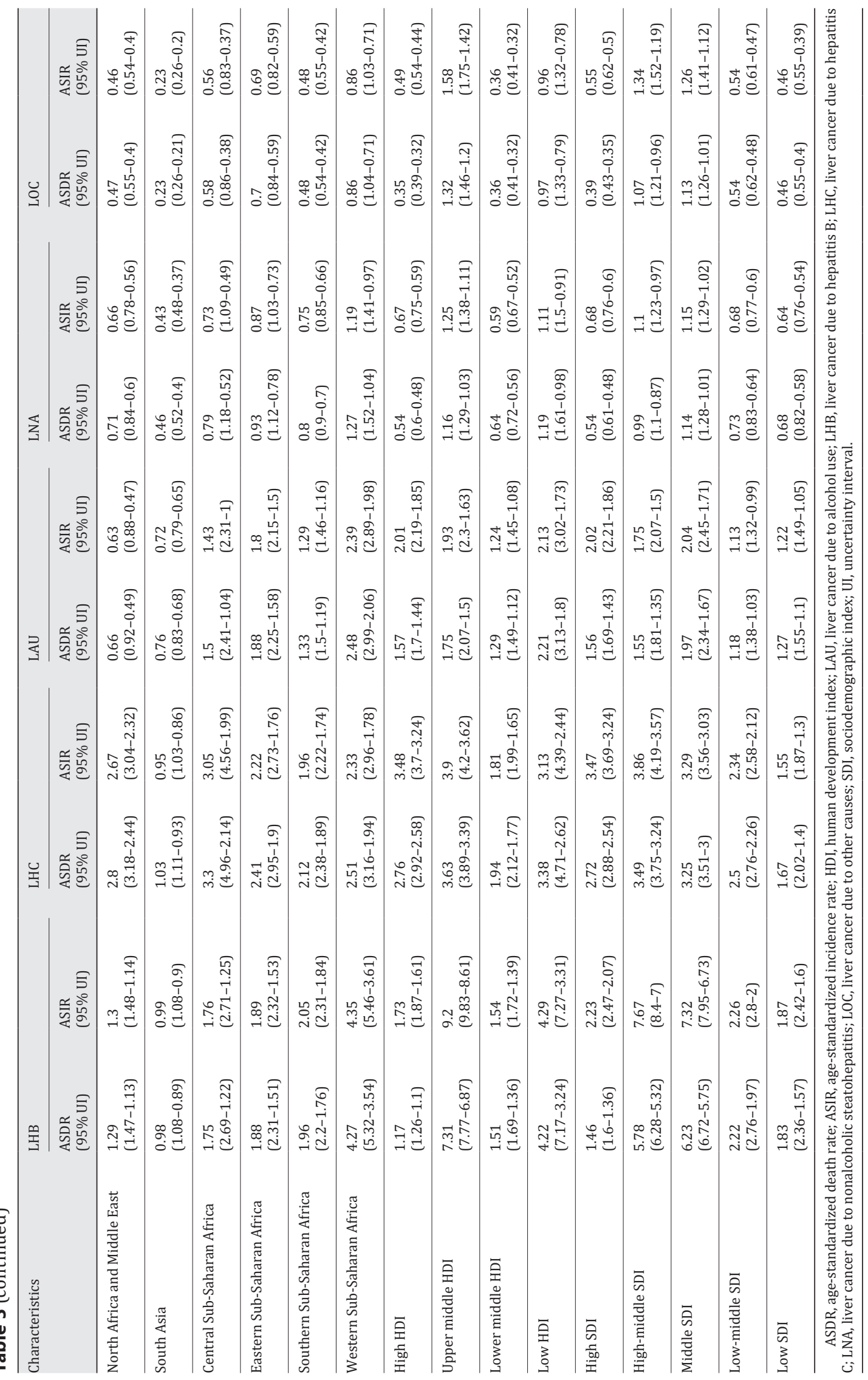


4.06]) of LAU among all 21 territories. In addition, $>30 \%$ of the total liver cancer-related deaths and incidents in Central Europe, Eastern Europe, Australasia, and Western Europe were caused by alcohol consumption. North Africa and the Middle East had the lowest LAU burden, and the ASDR and ASIR were $0.66(0.49-0.92)$ and 0.63 (0.47-0.88), respectively. Andean Latin America had the highest LNA- and LOC-related deaths (16.4 and 10.9\% of the total, respectively) and incidents ( 15.6 and $11.0 \%$ of the total, respectively). East Asia had the highest LNA and LOC burden; 39,912 (35,727-44,637) LOC-related deaths and 49,205 $(43,708-55,175)$ incidents were recorded, which accounted for 62.9 and $65.5 \%$ of the global LOC-related deaths and incidents, respectively.

HBV was the primary cause of liver cancer in the upper middle and low HDI regions, with respective ASIRs of 9.20 (8.61-9.83) and 7.67 (7.00-8.40), which accounted for 52.2 and $49.2 \%$, respectively, of the total liver cancer incidents in the two regions in 2017. HCV was the major cause of liver cancer and responsible for 43.9 and $45.1 \%$ of the total liver cancer incidents and deaths in the high HDI region. HBV was the primary cause of liver cancer in the high-middle and middle SDI regions. The ASDRs of LHB in these two regions (5.78 [5.32-6.28] and 6.23 [5.75-6.72], respectively) accounted for 45.6 and $47.1 \%$ of the total liver cancerrelated deaths, respectively, while the ASIRs (7.67 [7.00-8.40] and 7.32 [6.73-7.95], respectively) accounted for 49.2 and $50.2 \%$ of the total liver cancer incidents. HCV was the primary cause of liver cancer in the high SDI region, while LHC accounted for 41.4 and $43 \%$ of the total liver cancer incidents and deaths in this region, respectively. Alcohol use was another important cause of liver cancer in the high SDI region and was responsible for 23.3 and $22.7 \%$ of total liver cancer-related deaths and incidents.

\section{Liver Cancer Burden in Different Countries and Territories}

To further examine the regional differences in liver cancer burden, we analyzed the liver cancer incidence, mortality, and etiology in 195 countries and territories, as shown in Figures 2 and 3. The liver cancer burden was relatively high in China, North Korea, China Taiwan, Thailand, Tonga, Mongolia, Egypt, Mozambique, The Gambia, Mali, and Nigeria. The respective 2017 ASDRs for these countries were 21.30 (20.21-22.44), 25.91 (15.47-59.47), 25.09 (23.57-26.62), 22.65 (20.01-25.74), 24.65 (19.76-29.59), 100.14 (87.01-111.81), 20.47 (17.35-24.01), 30.51 (22.85-41.56), 39.20 (16.07-54.80), 32.22 (14.72-45.22), and 22.33 (7.90-87.92). China had the highest liver cancer incidents $(515,887[486,188-547,238])$ and deaths $(418,159$ [396,373-441,182]) in 2017, which accounted for 54.13 and $51.03 \%$ of the global liver cancer incidents and deaths, respectively. Furthermore, liver cancer-related deaths were also high in India, Japan, and the United States, and accounted for 4.33, 3.90, and $3.36 \%$ of the global, respectively. Compared to 1990, the ASDR and ASIR of liver cancer in 2017 increased by two-fold in Romania, Moldova, Australia, and the Netherlands, and the ASIR was increased by over one-fold in New Zealand, the United Kingdom, and the United States. In contrast, the ASDR and ASIR decreased by nearly or over $50 \%$ in Greece, Burkina Faso, and Sierra Leone.

The cause of liver cancer also varied greatly across countries and territories (as shown in online suppl. Fig. S1-10). LHB was most common in China, Thailand, Vietnam, South Korea, and India and accounted for $80 \%$ of the global LHB-related deaths in 2017. LHCrelated deaths were most frequent in China, Indonesia, Japan, Italy, the United States, Brazil, Egypt, and India, and the sum of these countries accounted for $>60 \%$ of the global. HBV was the primary cause of liver cancer in China, North Korea, Papua New Guinea, South Korea, Singapore, and Senegal and was responsible for $>50 \%$ of the total liver cancer incidents and deaths in these countries. On the other hand, HCV was the primary etiologic factor in Indonesia, Japan, Italy, Spain, Argentina, Chile, Uruguay, Mexico, Egypt, and Tunisia. LHC incidents and deaths were responsible for nearly or over $50 \%$ of the total 


\begin{tabular}{|c|c|c|}
\hline \multirow[b]{2}{*}{ Liver Cancer } & \multicolumn{2}{|l|}{ Liver Cancer 2020;9:563-582 } \\
\hline & DOI: $10.1159 / 000508568$ & $\begin{array}{l}\text { (O } 2020 \text { The Author(s). Published by S. Karger AG, Basel } \\
\text { www.karger.com/lic }\end{array}$ \\
\hline
\end{tabular}

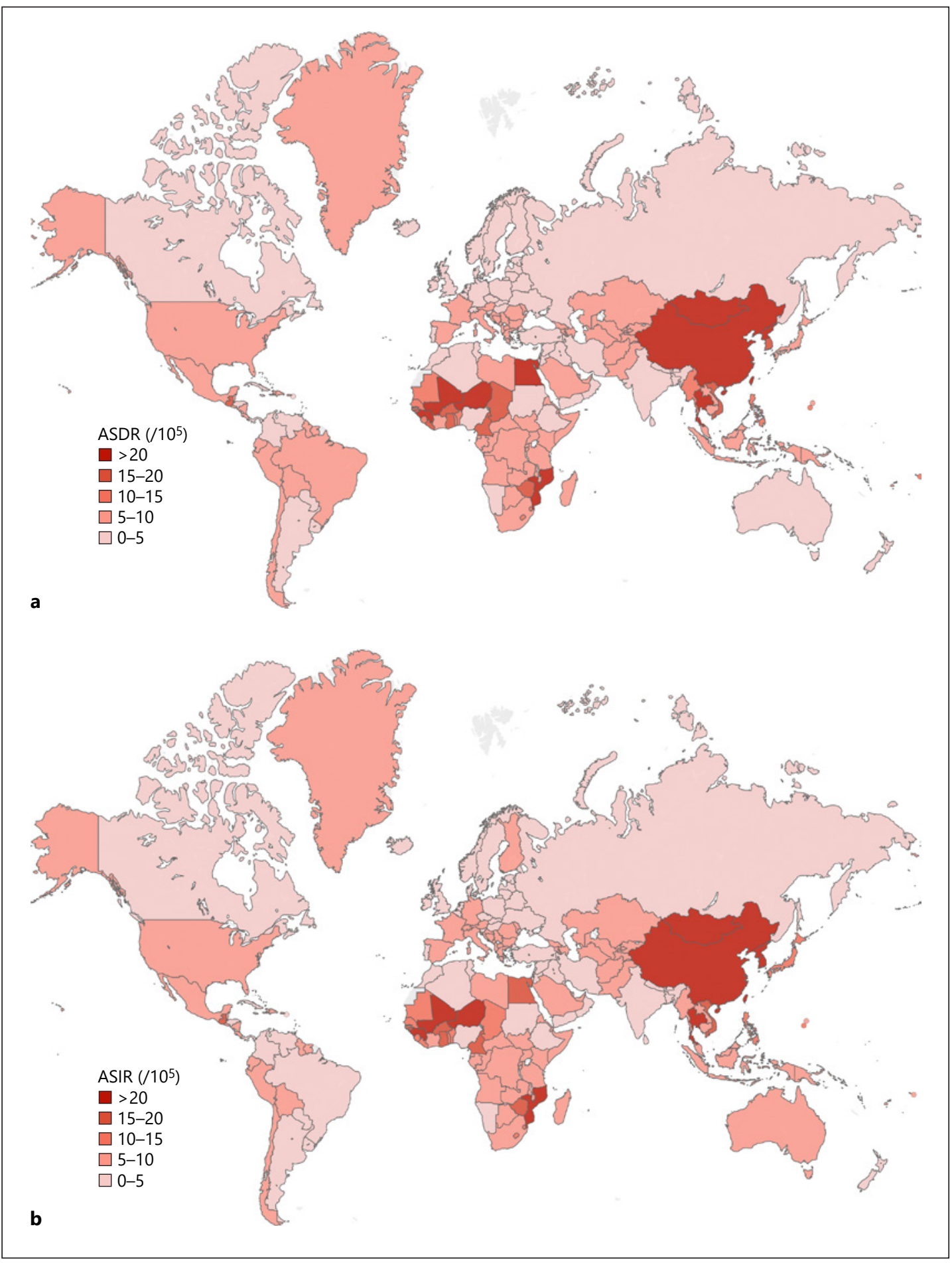

Fig. 2. Global disease burden of liver cancer in 195 countries and territories. a ASDR of liver cancer in 2017. b ASIR of liver cancer in 2017. ASDR, age-standardized death rate; ASIR, age-standardized incidence rate.

liver cancer incidents and deaths in these countries. Japan had the highest LHC incidents (65.77\% of total) and deaths (66.53\% of total), while LHB incidents and deaths only accounted for 9.74 and $9.15 \%$, respectively, of the respective regional rates. Alcoholism was the major cause of liver cancer in Bulgaria, Croatia, the Czech Republic, Hungary, 


\begin{tabular}{ll}
\cline { 2 - 2 } Liver Cancer 2020;9:563-582 \\
\cline { 2 - 2 } DOI: 10.1159/000508568 & $\begin{array}{l}\text { @) 2020 The Author(s). Published by S. Karger AG, Basel } \\
\text { www.karger.com/lic }\end{array}$ \\
\hline
\end{tabular}

Lin et al.: Global Burden of Primary Liver Cancer in 2017

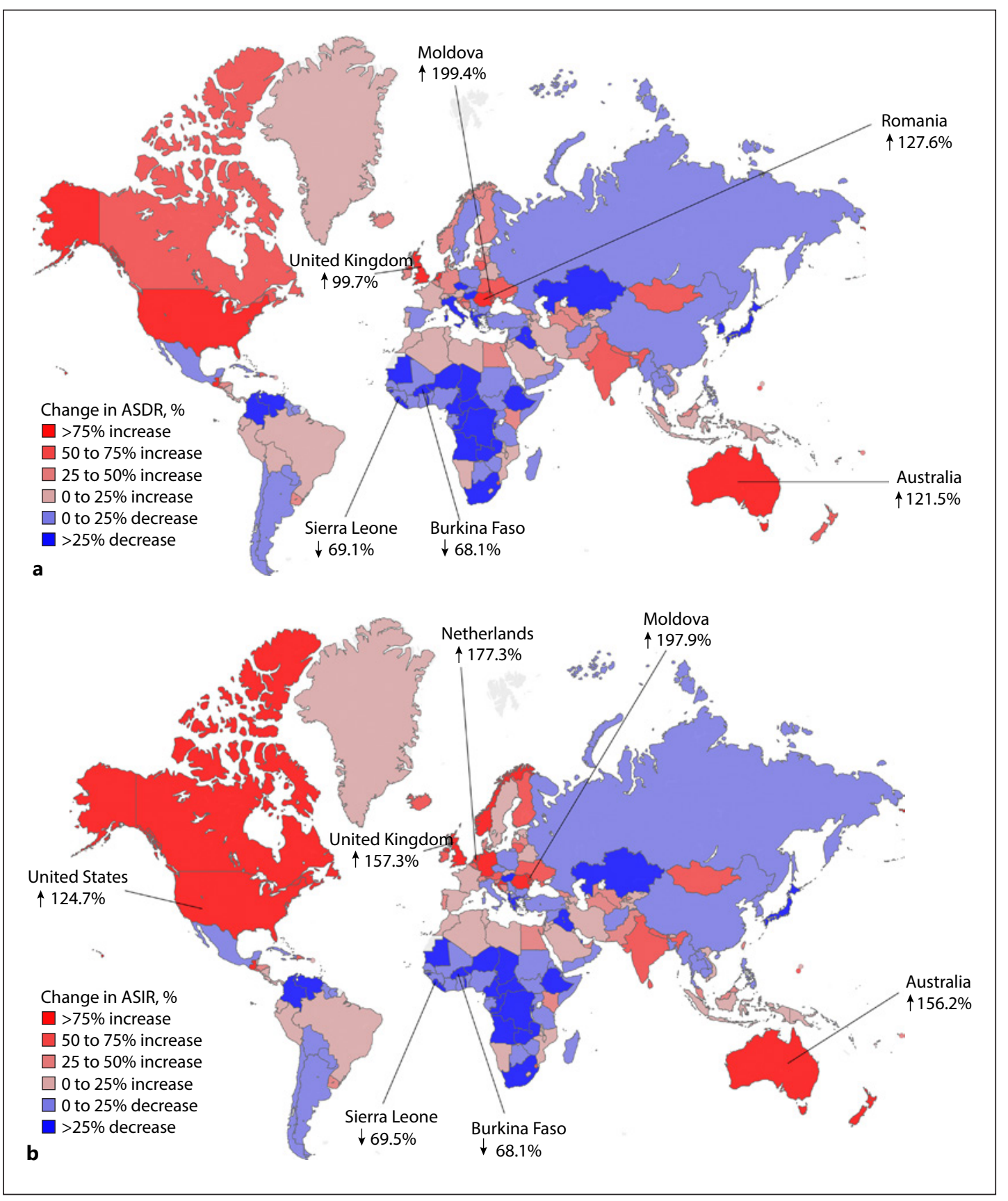

Fig. 3. Relative changes in ASDR (a) and ASIR (b) of liver cancer in 195 countries and territories between 1990 and 2017. ASDR, age-standardized death rate; ASIR, age-standardized incidence rate.

Poland, Slovakia, Austria, Denmark, Germany, Luxembourg, Sweden, Canada, and Greenland and accounted for $>40 \%$ of the cases and alcohol-related deaths in these countries. Canada had the highest LAU incidents (52.61\% of total) and deaths (52.37\% of total). LNA incidents were relatively low in every country and were responsible for $15 \%$ of the total liver cancer-related deaths in only 12 countries, with the highest recorded in Iran $(20.58 \%)$. Other causes were the least common factors, and LOC incidents and deaths were generally $<10 \%$ in most countries. Sudan had the highest LOC incidents and deaths, accounting for 


\begin{tabular}{l|l}
\hline Liver Cancer 2020;9:563-582 \\
\hline DOI: 10.1159/000508568 & $\begin{array}{l}\text { ( 2 2020 The Author(s). Published by S. Karger AG, Basel } \\
\text { www.karger.com/lic }\end{array}$ \\
\hline
\end{tabular}

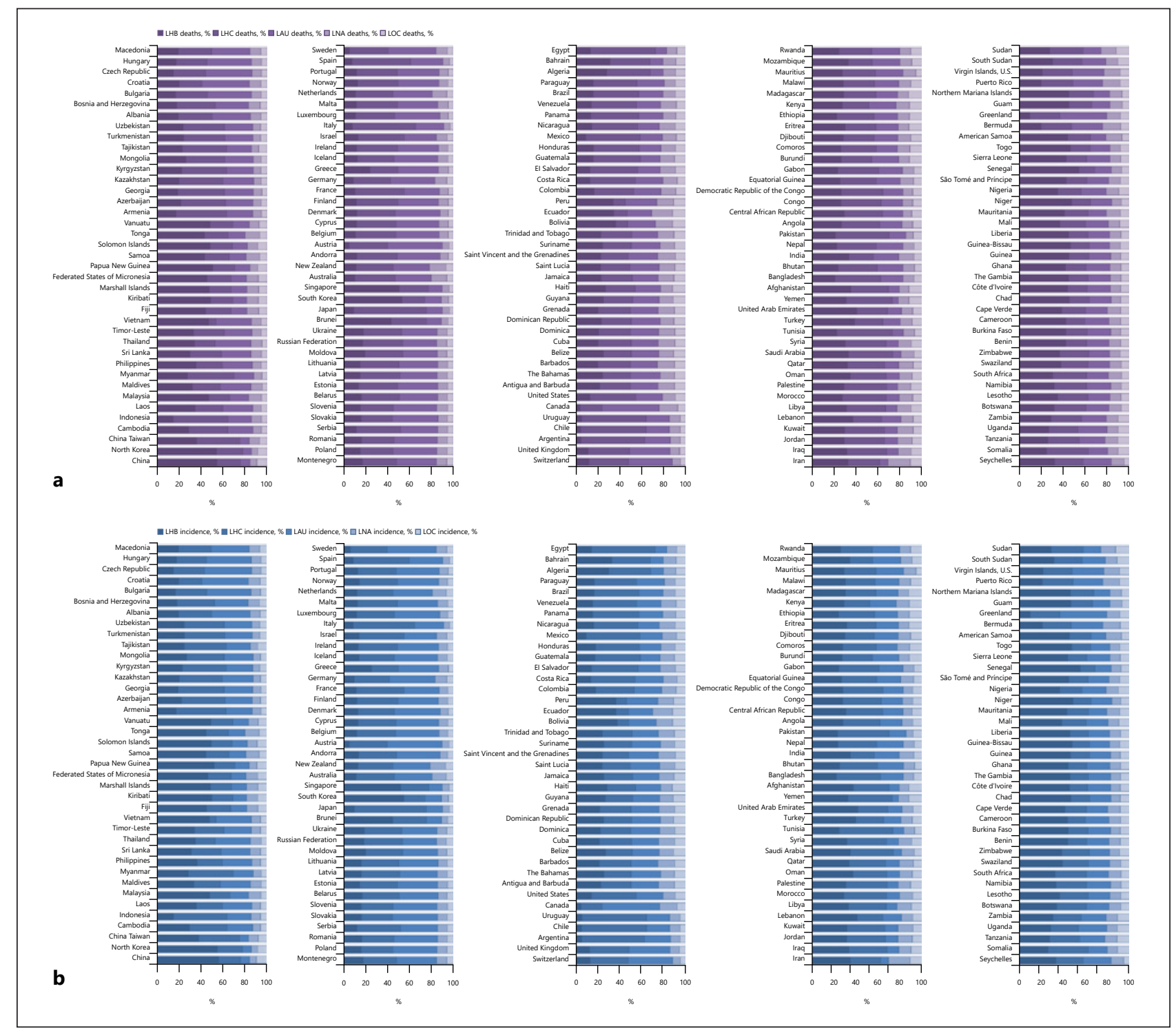

Fig. 4. Proportion of five etiologies of liver cancer death cases (a) and incidence cases (b) in 2017. LAU, liver cancer due to alcohol use; LHB, liver cancer due to hepatitis B; LHC, liver cancer due to hepatitis C; LNA, liver cancer due to nonalcoholic steatohepatitis; LOC, liver cancer due to other causes.

11.69 and $12.12 \%$ of the total liver cancer incidents and deaths in the country, respectively. The proportion of five etiologies of liver cancer death and incidence cases in different countries and territories is shown in Figure 4.

\section{Sex-Related Differences in Liver Cancer Burden}

Liver cancer incidence and mortality were approximately three times higher in males than in females (Fig. 5a; online suppl. Fig. S11-15). The total number of liver cancer-related deaths in 2017 was 572,128 (543,395-609,965) for males and 247,308 (238,516-257,298) for females, although the incidence and mortality rates in the two sexes differed across regions. East Asia had the highest ASDR and ASIR for either sex, and the latter was as high as 40.4 (37.54-44.08) among males. The ASDRs and ASIRs were similar for both sexes in Andean Latin America, Oceania, the Caribbean, and Central Latin America. The greatest difference in 
the ASDR of males (16.32 [14.68-17.59]) and females (5.16 [4.48-5.67]) was seen in highincome Asia Pacific.

The etiologies of liver cancer exhibited differences between males and females. Newlydiagnosed LHB and LAU cases and deaths were more common among males. The 2017 global ASDR and ASIR of LHB were, respectively, 6.43 (5.99-7.02) and 8.09 (7.51-8.77) in males and only $1.72(1.6-1.86)$ and $1.96(1.82-2.11)$ in females. Similarly, the global ASDR and ASIR of LAU were higher among males at $2.72(2.42-3.1)$ and 3.05 (2.7-3.48), respectively, compared to 0.65 (0.57-0.74) and $0.67(0.59-0.76)$ in females. The ASDR and ASIR of LOC in females were generally $60 \%$ of those in males. The etiologic factors also demonstrated region-specific characteristics between males and females. While the ASDR and ASIR of LHB were similar between the sexes in Andean Latin America, those of LHC, LNA, and LOC were significantly higher among females. For example, the ASDR of LOC in this region was 1.07 (0.91-1.25) in females and $0.49(0.41-0.58)$ in males.

\section{Age-Related Differences in Liver Cancer Burden}

Liver cancer burden was the highest among individuals older than 50 years and has been gradually increasing annually (results are shown in Fig. 5b and online suppl. Fig. S16-20). While the $50+$ age group accounted for $>80 \%$ of total liver cancer incidents and deaths in

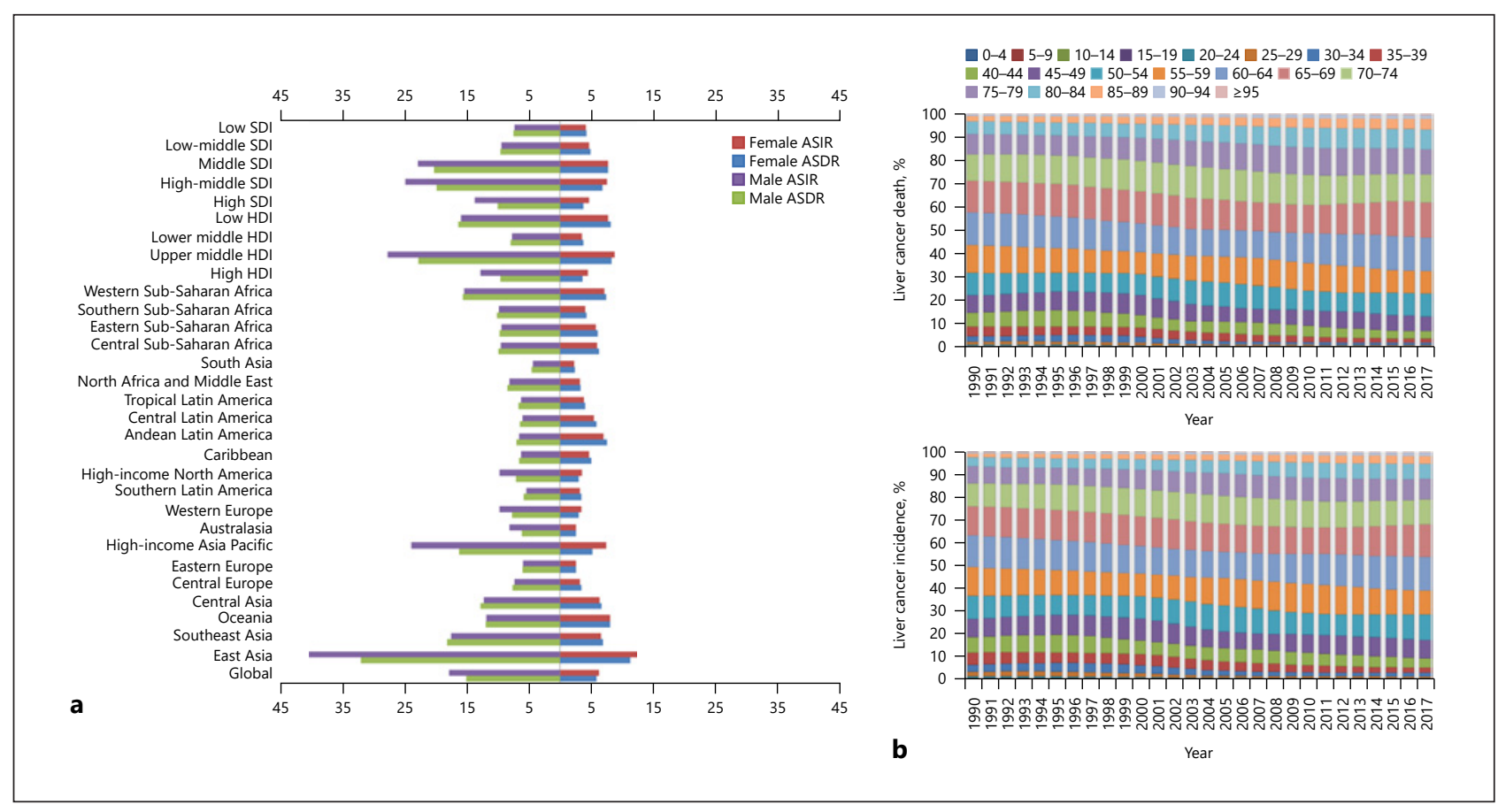

Fig. 5. a Age-standardized global deaths and incidence of liver cancer in 2017 by sex. b Age-specific global contributions to incidence and mortality of liver cancer in 2017. ASDR, age-standardized death rate; ASIR, age-standardized incidence rate; HDI, human development index; SDI, sociodemographic index.

Fig. 6. Trends in global liver cancer from 1990 to 2017. ASDR of males (a), ASIR of males (b), ASDR of females (c), ASIR of females (d), and global average age of onset of liver cancer and five etiologies from 1990 to 2017 (e). * The APC is significantly different from zero at an alpha of 0.05. APC, annual percentage change; ASDR, age-standardized death rate; ASIR, age-standardized incidence rate; LAU, liver cancer due to alcohol use; LHB, liver cancer due to hepatitis B; LHC, liver cancer due to hepatitis C; LNA, liver cancer due to nonalcoholic steatohepatitis; LOC, liver cancer due to other causes.

(For figure see next page.)

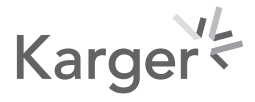




\begin{tabular}{|c|c|c|}
\hline \multirow[b]{2}{*}{ Liver Cancer } & \multicolumn{2}{|l|}{ Liver Cancer 2020;9:563-582 } \\
\hline & DOI: $10.1159 / 000508568$ & $\begin{array}{l}\text { () } 2020 \text { The Author(s). Published by S. Karger AG, Basel } \\
\text { www.karger.com/lic }\end{array}$ \\
\hline
\end{tabular}
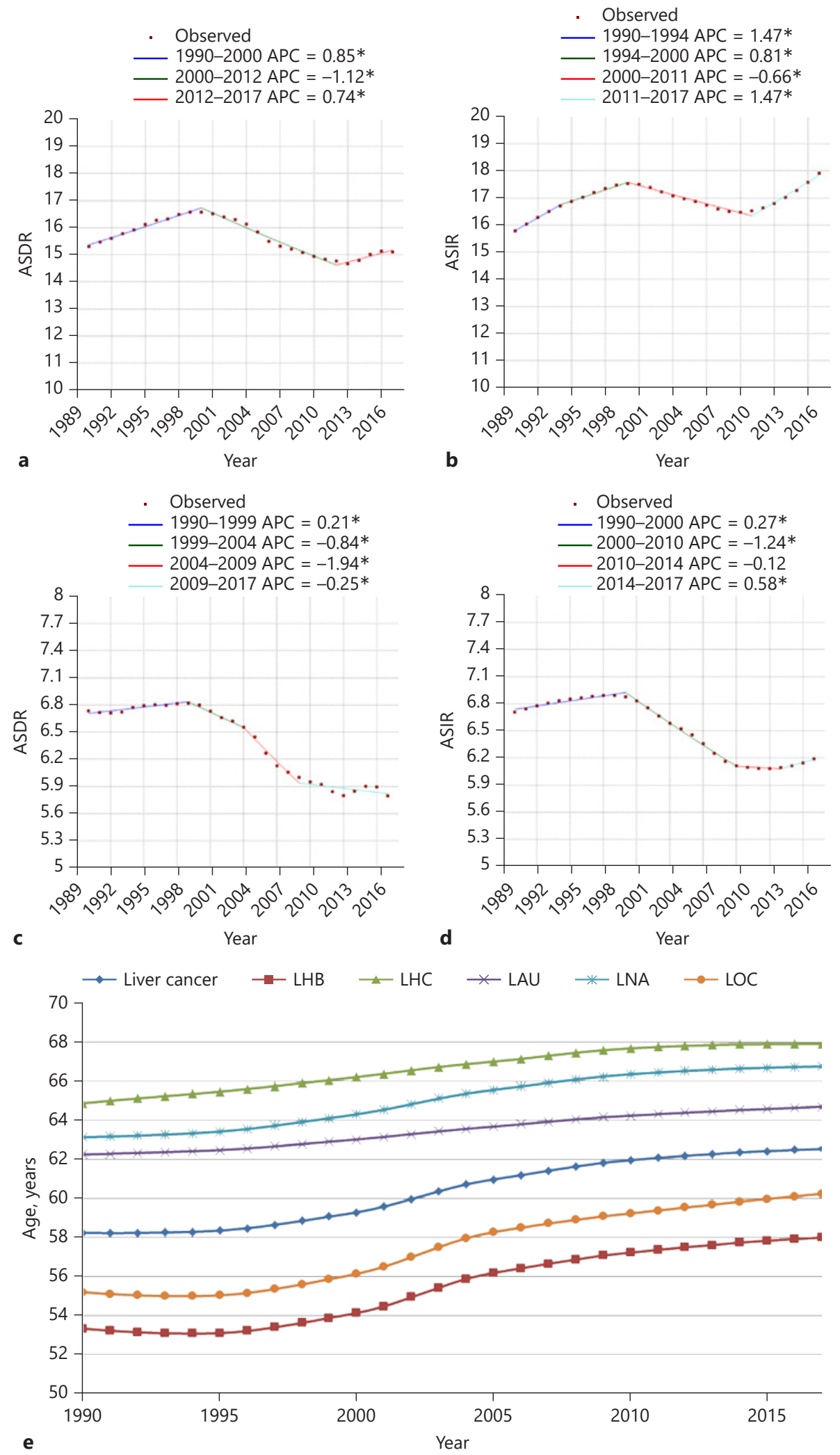
$1990,<1 \%$ of the same was recorded among individuals $<20$ years of age in the same year and has been decreasing over time. Different liver cancer etiologies also demonstrated agerelated preferences. Although LHB incidents and deaths were most prevalent in the 50+ population, they remained $<80 \%$ of the total liver cancer incidents and deaths over the last 28 years. The primary cause of liver cancer in the $\leq 20$ age group was HBV, and LHB accounted for 68 and $68.2 \%$, respectively, of the total liver cancer-related deaths and incidents in this population in 2017. LHC and LNA were most prevalent in the 50+ age group and accounted for $>90 \%$ and even up to $95 \%$ of the total liver cancer-related deaths in recent years. LAU and LNA were not observed in the $\leq 15$ age group, wherein HBV, HCV, and other causes were the common etiologies.

Furthermore, the mean onset age of liver cancer was 58.2 years in 1990, which increased to 62.5 years in 2017, and also differed depending on the etiology. The mean onset ages of LHB, LHC, LAU, LNA, and LOC were respectively $53.3,64.9,62.2,63.1$, and 55.2 years, respectively, in 1990 and increased to 58.0, 67.9, 64.7, 66.8, and 60.2, respectively, in 2017. While LHB and LOC patients were younger, both showed a greater increase in mean onset age compared to the LHC, LAU, and LNA patients, with the greatest increase (nearly 5 years) seen in LOC (as shown in Fig. 6e).

\section{Analysis of Liver Cancer Trends}

We analyzed the trends of global liver cancer incidence and mortality from 1990 to 2017 and found that both the ASDR and ASIR increased at the beginning and end of the 28-year period, but decreased in the interim [10]. Interestingly, the ASDR and ASIR trends were different between males and females, as shown in Figure 6. Both showed increasing trends at the beginning and end of the 28-year period in the male patients. The ASDR in females showed a decreasing trend most of the time. It slowly increased from 1990 to 1999 (APC $=0.2$ ), rapidly declined until 2012, and remained stable from 2012 to 2017. The etiology of liver cancer also affected the time-dependent change in its trends. The ASDR and ASIR of LHB showed similar trends from 1990 to 2017: both increased from 1990 to 2000 (APC $=0.54$ and 0.69, respectively) and then rapidly decreased at an APC of -1.5 in the subsequent decade. However, ASIR was increased at an APC of 1.42 from 2012 until 2017. The ASIR of LHB in 2017 (4.93 [4.625.29]) was similar to that in 1990 (4.93 [4.51-5.42]), but the ASDR was far lower in 2017 (3.99 [3.74-4.27]) than in 1990 (4.64 [4.24-5.1]). The ASDR and ASIR of LHC also followed a similar "increase-decrease-increase" pattern. The ASDR of LAU fluctuated minimally (1.581.70 ) in the past 30 years, but the ASIR increased by nearly 12\% from 1990 (1.6 [1.41.86]) to 2017 (1.79 [1.59-2.05]). The ASDR and ASIR of LNA showed the most rapid increase $(\mathrm{APC}=2.08)$ between 1990 and 2017 compared to the other etiologic types, increasing respectively from $0.71(0.62-0.81)$ and $0.69(0.61-0.79)$ in 1990 to $0.85(0.75-0.94)$ and 0.91 (0.81-1) in 2017. Finally, the ASDR and ASIR of LOC followed a similar "increase-decreaseincrease" trend as that of LHB. Its ASIR had an APC of 1.96 between 2014 and 2017 and was significantly higher in 2017 than in 1990. The trends of five etiologies of liver cancer are shown in online supplementary Figures S21-25.

\section{Discussion}

In this study, we evaluated the global liver cancer burden in 2017 demarcated by etiology, sex, age, socioeconomic region, and country. Consistent with previous findings, our results showed that liver cancer is one of the major causes of cancer-related deaths globally, and its cause varies across regions and countries [7, 8]. However, previous studies mainly focused on analyzing overall liver cancer incidence, whereas we performed a comprehensive analysis

\section{Karger's}


Lin et al.: Global Burden of Primary Liver Cancer in 2017

of the causes of liver cancer in the different sexes, age groups, regions, and countries in terms of incidence, mortality, and number of incidents and deaths. In addition, we also examined the trends in the incidence and mortality of liver cancer with different etiologies over the last 30 years.

Liver cancer is associated with HBV and HCV infections, alcohol consumption, NASH, and other factors. The global liver cancer incidence and the number of liver cancer cases have significantly increased from 1990 to 2017 and are mainly attributed to the LHB, LHC, and LAU. In particular, HCV is the major driver of the increase in global liver cancer incidence. Since the incidence, mortality, and etiology of liver cancer differ across regions, age groups, and sexes, understanding the specific etiologic patterns is essential for its prevention. HBV and HCV are at present the primary causes of liver cancer and were responsible for nearly $70 \%$ of the global liver cancer-related deaths and incidents in 2017. LHB has a distinct regional preference and is most common in East Asia, Southeast Asia, and South Asia, the three regions accounting for about $85 \%$ of the global LHB incidents and deaths, with a major contribution from China. However, LHB incidence and mortality in China have significantly decreased from 1990 to 2017, mainly due to HBV immunization in newborns and the implementation of HBV screening programs [11]. HCV infection and alcohol abuse are the main risk factors for liver cancer in the high SDI and high HDI regions, such as the United Kingdom, the United States, Italy, and Canada, which are the main contributors to the increase in global LHC incidence. HCV can be transmitted by drug injection, transfusion of contaminated blood, or use of contaminated needles [12-14]. The emergence of direct-acting antivirals in recent years has revolutionized HCV treatment and effectively controlled LHC incidence and mortality. Alcohol can induce liver cirrhosis and create an environment conducive to HBV infection $[15,16]$. In fact, the risk of liver cancer is doubled in individuals who have consumed $>80 \mathrm{~g}$ of alcohol for $>10$ years [17]. The LAU burden is higher in Central Europe, Eastern Europe, Australasia, and Western Europe. Aflatoxin $B_{1}$ intake is also an important cause of liver cancer. It is primarily produced by Aspergillus flavus and Aspergillus parasiticus and is often present in grains, nuts, and oils harvested in tropical and subtropical regions [18-20]. The risk of liver cancer due to aflatoxin $\mathrm{B}_{1}$ consumption is higher in India $[21,22]$.

The significant differences in liver cancer incidence and mortality between males and females can be attributed to HBV infection and different lifestyles (such as alcohol consumption and smoking). Interestingly, the sex difference in liver cancer incidence and mortality is less in the lower middle and low HDI regions as well as in the low-middle and low SDI regions. The liver cancer incidence and mortality is higher in females than in males in the Latin American regions, especially in Andean Latin America, although the underlying cause needs to be investigated further. Analysis of the trends from 1990 to 2017 showed that the incidence and mortality of liver cancer has increased in males but decreased in females, indicating that this gap may widen in the foreseeable future. The global liver cancer burden is highest among individuals $>50$ years of age, and this population is the source of $95 \%$ of the LHC and LNA burden, which is higher than for the other etiologies. In addition, we found that the mean onset age of liver cancer due to various causes has been gradually increasing. As the global population ages, the liver cancer burden will continue to increase among the elderly demography, thus calling for better preventive measures. Furthermore, HBV is the major cause of liver cancer in those aged $<20$ years, which underscores the importance of immunizing children against HBV. A previous report stated that children immunized with the hepatitis B vaccine still have a risk of developing chronic hepatitis after 10 years, indicating that booster doses are needed to maintain the immune response [23].

LNA mortality and incidence have been rapidly increasing in the last 30 years. NASH is an advanced form of NAFLD that can lead to cirrhosis and subsequently liver cancer. It is often accompanied by obesity, diabetes, and excessive iron deposition, which may be high risk

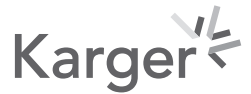


factors for the progression of NASH to liver cancer [24-26]. Although the mortality due to LHB, LHC, LAU, and LOC has gradually decreased in the last few years and showed an overall decreasing trend from 1990 to 2017, the incidence of these liver cancers has rapidly risen in recent years. Our analysis suggests that the liver cancer burden may continue to increase, and therefore the preventive and treatment measures for each etiologic type must be improved.

The primary preventive measures for reducing the global liver cancer burden [27] include (1) strengthening nosocomial infection control and implementing stringent HBsAg and HBV DNA screening, (2) improving the level of hepatitis B immunization in newborns, infants, unimmunized children $<15$ years of age, and high-risk individuals, (3) immunizing infants born to HBV-infected women with hepatitis B vaccine and hepatitis B immunoglobulin and providing antiviral intervention such as oral nucleoside (nucleotide) analogues to pregnant women with high viremia in their late pregnancy, (4) improving food preservation processes to prevent fungal contamination and reduce aflatoxin $\mathrm{B}_{1}$ levels, and (5) avoiding other environmental and behavioral risk factors like microcystins, smoking, alcohol consumption, obesity, and diabetes. Secondary preventive measures, such as early liver cancer screening, should also be implemented to detect precancerous lesions and prevent cancer progression. The frequency of liver cancer screening should be increased among the elderly and high-risk populations. Common screening methods include detection of serum tumor markers (alphafetoprotein) and ultrasound examination. Computed tomography, magnetic resonance imaging, angiography, and radionuclide scanning may also be used if necessary. Finally, tertiary prevention measures should be emphasized to reduce local recurrence and metastasis in patients after recovery. Recurrence after remission of HBV-positive liver cancer can generally be prevented by antivirals, and new lesions can be treated by inhibiting the vascular endothelial growth factor receptor and other proangiogenic factors [28]. However, since the efficacy of systemic liver cancer treatment is still not satisfactory, novel therapeutic drugs and methods are urgently needed. In addition, traditional herbs should also receive attention in the treatment of primary liver cancer, such as traditional Chinese medicine. Studies have confirmed that some Chinese herbal compounds and monomeric compounds in traditional Chinese medicine have better anti-hepatocarcinogenic effects [29], such as curcumin, triptolide, oridonin, emodin, matrine, etc. [30,31]. These ingredients may be potential drugs for treating liver cancer.

\section{Conclusions}

The continuous rise in global liver cancer burden requires collaborative prevention and control from the government, multilevel prevention and control departments, cancer centers, and hospitals. In addition to reinforcing hepatitis B vaccination in newborns, the management of individuals who are at high risk of HBV infection should also be improved, along with greater prevention of HCV infection and alcohol restriction. Furthermore, health management and early screening should be strongly enforced for individuals who are at high risk of developing liver cancer, and research into identifying novel liver tumor markers should be encouraged to improve the early diagnosis rate. By analyzing the burden of liver cancer with different etiologies at the sex, age, regional, and national levels, our study will help elucidate the characteristics of global liver cancer burden and establish more effective and targeted prevention strategies.

\section{Limitations}

Although the GBD estimates can fill in the missing or unavailable data in disease burden, some limitations are still worth noting. (1) The accuracy of the GBD estimates is mostly 
dependent on the quality and quantity of the data. These estimates have a high level of uncertainty due to the low proportions of South Americans, Asians, and Africans covered by highquality cancer registries. (2) The increasing trends in liver cancer burden are associated with the increase in cancer registry sites. (3) Underreporting and misdiagnosis are common during cancer registration, especially in developing countries. (4) The main issues with data quality in the estimation of liver cancer burden are the misdiagnosis of liver metastases as primary liver cancer and the underreporting of liver cancer on death certificates, which could be the result of poor diagnostic ability. (5) When determining the cause of liver cancer, HBV and HCV can be objectively classified according to laboratory evaluations, but other factors such as alcohol consumption can only be self-reported by the patients. (6) The liver cancer etiologies might estimate errors if multiple causes are present.

\section{Acknowledgements}

We appreciate the works by the GBD 2017 study collaborators. The datasets generated and/or analyzed during the current study are available in the GBD repository (http://ghdx.healthdata.org/gbd-results-tool).

\section{Statement of Ethics}

This study is an analysis of the burden of liver cancer and the data were obtained from the GBD database, so ethics approval and consent to participate were not necessary for this paper.

\section{Conflict of Interest Statement}

The authors declare no conflict of interest regarding the content of this paper.

\section{Funding Sources}

This study was financially supported by the Fundamental Research Funds for the Central Public Welfare Research Institutes (ZXKT17013, ZZ11-092, and ZZ13-YQ-059) and the Young Elite Scientists Sponsorship Program by CACM(2018QNRC2-C01).

\section{Author Contributions}

L. Lin, J. Ni, and L. Yan performed the study design and data collection and wrote the manuscript. L. Lin, L. Yan, and C. Qu analyzed the data and interpreted the results. L. Lin and Y. Liu revised the manuscript. J. Ni and $\mathrm{H}$. Li reviewed and finalized the manuscript and conceptualized the study. All authors read and approved the final manuscript.

\section{References}

1 Bray F, Ferlay J, Soerjomataram I, Siegel RL, Torre LA, Jemal A. Global cancer statistics 2018: GLOBOCAN estimates of incidence and mortality worldwide for 36 cancers in 185 countries. CA Cancer J Clin. 2018 Nov;68(6): 394-424.

2 National Cancer Institute. Surveillance, Epidemiology, and End Results Program. Available at: https://seer. cancer.gov/statfacts/html/livibd.html [accessed September 2019].

3 Allemani C, Matsuda T, Di Carlo V, Harewood R, Matz M, Nikšić M, et al.; CONCORD Working Group. Global surveillance of trends in cancer survival 2000-14 (CONCORD-3): analysis of individual records for 37513025 patients diagnosed with one of 18 cancers from 322 population-based registries in 71 countries. Lancet. 2018 Mar;391(10125):1023-75. 
4 El-Serag HB. Epidemiology of viral hepatitis and hepatocellular carcinoma. Gastroenterology. 2012 May; 142(6):1264-73.e1.

5 Ascha MS, Hanouneh IA, Lopez R, Tamimi TA, Feldstein AF, Zein NN. The incidence and risk factors of hepatocellular carcinoma in patients with nonalcoholic steatohepatitis. Hepatology. 2010 Jun;51(6):1972-8.

6 Zheng C, Zeng H, Lin H, Wang J, Feng X, Qiu Z, et al. Serum microcystin levels positively linked with risk of hepatocellular carcinoma: A case-control study in southwest China. Hepatology. 2017 Nov;66(5):1519-28.

7 Akinyemiju T, Abera S, Ahmed M, Alam N, Alemayohu MA, Allen C, et al.; Global Burden of Disease Liver Cancer Collaboration. The Burden of Primary Liver Cancer and Underlying Etiologies From 1990 to 2015 at the Global, Regional, and National Level: Results From the Global Burden of Disease Study 2015. JAMA Oncol. 2017 Dec;3(12):1683-91.

8 Liu Z, Jiang Y, Yuan H, Fang Q, Cai N, Suo C, et al. The trends in incidence of primary liver cancer caused by specific etiologies: results from the Global Burden of Disease Study 2016 and implications for liver cancer prevention. J Hepatol. 2019 Apr;70(4):674-83.

9 Global Burden of Disease Collaborative Network. Global Burden of Disease Study 2017 (GBD 2017) Results. Seattle, United States: Institute for Health Metrics and Evaluation (IHME), 2018. Available at: http://ghdx. healthdata.org/gbd-results-tool.

10 Lin L, Yan L, Liu Y, Yuan F, Li H, Ni J. Incidence and death in 29 cancer groups in 2017 and trend analysis from 1990 to 2017 from the Global Burden of Disease Study. J Hematol Oncol. 2019 Sep;12(1):96.

11 Chen W, Zheng R, Baade PD, Zhang S, Zeng H, Bray F, et al. Cancer statistics in China, 2015. CA Cancer J Clin. 2016 Mar-Apr;66(2):115-32.

12 Bosetti C, Levi F, Boffetta P, Lucchini F, Negri E, La Vecchia C. Trends in mortality from hepatocellular carcinoma in Europe, 1980-2004. Hepatology. 2008 Jul;48(1):137-45.

13 El-Serag HB. Epidemiology of hepatocellular carcinoma in USA. Hepatol Res. 2007 Sep;37(Suppl 2):S88-94.

14 Denniston MM, Jiles RB, Drobeniuc J, Klevens RM, Ward JW, McQuillan GM, et al. Chronic hepatitis C virus infection in the United States, National Health and Nutrition Examination Survey 2003 to 2010. Ann Intern Med. 2014 Mar;160(5):293-300.

15 Rehm J, Samokhvalov AV, Shield KD. Global burden of alcoholic liver diseases. J Hepatol. 2013 Jul;59(1):160-8.

16 Marcellin P, Pequignot F, Delarocque-Astagneau E, Zarski JP, Ganne N, Hillon P, et al. Mortality related to chronic hepatitis B and chronic hepatitis C in France: evidence for the role of HIV coinfection and alcohol consumption. J Hepatol. 2008 Feb;48(2):200-7.

17 Morgan TR, Mandayam S, Jamal MM. Alcohol and hepatocellular carcinoma. Gastroenterology. 2004 Nov; 127(5 Suppl 1):S87-96.

18 Bankole SA, Ogunsanwo BM, Mabekoje 00. Natural occurrence of moulds and aflatoxin B1 in melon seeds from markets in Nigeria. Food Chem Toxicol. 2004 Aug;42(8):1309-14.

19 Prado G, Altoé AF, Gomes TC, Leal AS, Morais VA, Oliveira MS, et al. Occurrence of aflatoxin B1 in natural products. Braz J Microbiol. 2012 Oct; 43(4):1428-36.

20 Maringe DT, Chidewe C, Benhura MA, Mvumi BM, Murashiki TC, Dembedza MP, et al. Natural postharvest aflatoxin occurrence in food legumes in the smallholder farming sector of Zimbabwe. Food Addit Contam Part B Surveill. 2017 Mar;10(1):21-6.

21 Asim M, Sarma MP, Kar P. Etiological and molecular profile of hepatocellular cancer from India. Int J Cancer. 2013 Jul;133(2):437-45.

22 Asim M, Sarma MP, Thayumanavan L, Kar P. Role of aflatoxin B1 as a risk for primary liver cancer in north Indian population. Clin Biochem. 2011 Oct;44(14-15):1235-40.

23 Wang Y, Chen T, Lu LL, Wang M, Wang D, Yao H, et al. Adolescent booster with hepatitis B virus vaccines decreases HBV infection in high-risk adults. Vaccine. 2017 Feb;35(7):1064-70.

24 Michelotti GA, Machado MV, Diehl AM. NAFLD, NASH and liver cancer. Nat Rev Gastroenterol Hepatol. 2013 Nov;10(11):656-65.

25 Yasui K, Hashimoto E, Komorizono Y, Koike K, Arii S, Imai Y, et al. Characteristics of patients with nonalcoholic steatohepatitis who develop hepatocellular carcinoma. Clin Gastroenterol Hepatol. 2011 May;9(5):428-33; quiz e50.

26 Sorrentino P, D’Angelo S, Ferbo U, Micheli P, Bracigliano A, Vecchione R. Liver iron excess in patients with hepatocellular carcinoma developed on non-alcoholic steato-hepatitis. J Hepatol. 2009 Feb;50(2):351-7.

27 Giacomin A, Cazzagon N, Sergio A, Vanin V, Farinati F. Hepatitis B virus-related hepatocellular carcinoma: primary, secondary, and tertiary prevention. Eur J Cancer Prev. 2011 Sep;20(5):381-8.

28 Scartozzi M, Faloppi L, Svegliati Baroni G, Loretelli C, Piscaglia F, Iavarone M, et al. VEGF and VEGFR genotyping in the prediction of clinical outcome for HCC patients receiving sorafenib: the ALICE-1 study. Int J Cancer. 2014 Sep;135(5):1247-56.

29 Li X, Yang G, Li X, Zhang Y, Yang J, Chang J, et al. Traditional Chinese medicine in cancer care: a review of controlled clinical studies published in Chinese. PLoS One. 2013;8(4):e60338.

30 Li SG, Shi QW, Yuan LY, Qin LP, Wang Y, Miao YQ, et al. C-Myc-dependent repression of two oncogenic miRNA clusters contributes to triptolide-induced cell death in hepatocellular carcinoma cells. J Exp Clin Cancer Res. 2018 Mar;37(1):51.

31 Hu Y, Wang S, Wu X, Zhang J, Chen R, Chen M, et al. Chinese herbal medicine-derived compounds for cancer therapy: a focus on hepatocellular carcinoma. J Ethnopharmacol. 2013 Oct;149(3):601-12. 